\title{
Nanoscale neuroimaging
}

DOI:

10.1038/1935
Reporting in Nature, Stefan Hell, Reinhard Jahn and colleagues provide new insights into the process of synaptic vesicle recycling. They used an innovative method - known as stimulated emission depletion (STED) microscopy - which overcomes the resolution limitations of
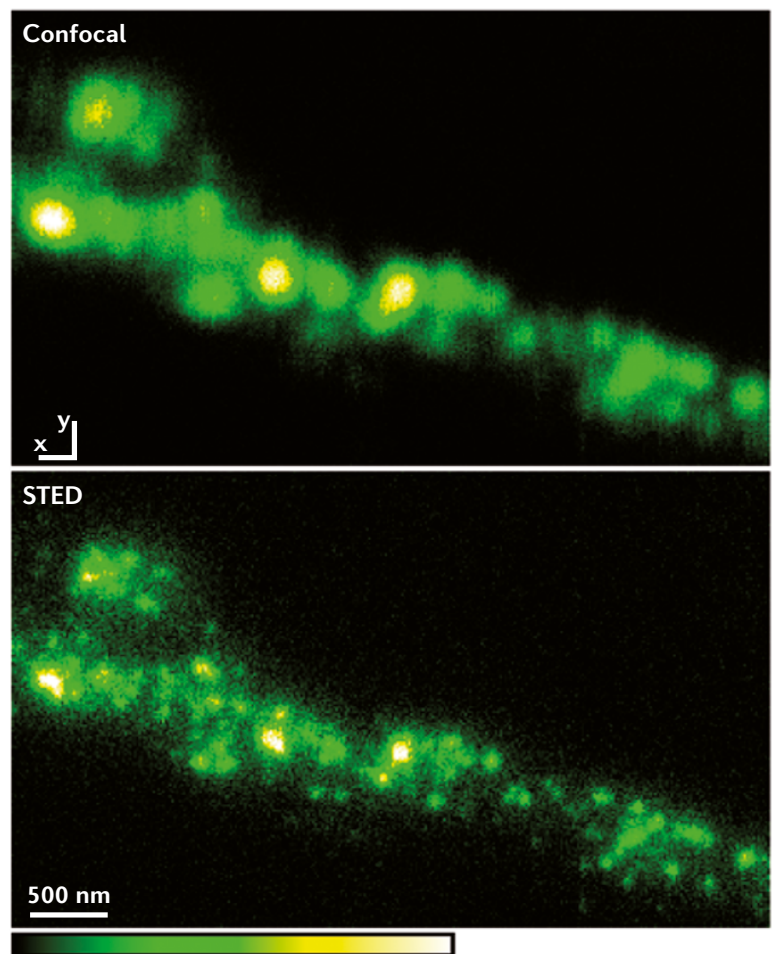

Cultured hippocampal neurons labelled with an antibody specific for synaptotagmin, followed by permeabilization and fixation. Images show a comparison between confocal (top) and STED (bottom) resolution. Images courtesy of R. Jahn, Max Planck Institute for Biophysical Chemistry, Göttingen, Germany. traditional fluorescence microscopy methods and allows cellular structures, such as synaptic vesicles, to be viewed with nanoscale resolution.

Until now, optical microscopy techniques have been limited to observing structures at a resolution that corresponds to half the shortest wavelength of visible light $(\sim 200$ $\mathrm{nm})$. Hell and co-workers have developed the STED technique to override this 'diffraction barrier'. They use a doughnut-shaped beam (the STED beam) in addition to an excitation beam to restrict the fluorescence by an order of magnitude below the diffraction limit to a focal point in the centre of the excitation beam. This results in the sharpening of images to a resolution of 45-70 $\mathrm{nm}$, thereby allowing synaptic vesicles to be seen.

Hell, Jahn and co-workers have used STED for the first time in the CNS to address an enduring question relating to synaptic vesicle recycling during synaptic transmission. It is well known that after synaptic vesicles have fused with the cell membrane to release neurotransmitter they are then recycled back into the cell and refilled with neurotransmitter. But until now, it has not been possible to determine precisely what happens to vesicles after neurotransmitter release. Do the components of the vesicle membrane remain together after exocytosis or do they fuse on the plasma membrane? Current thinking suggests that these components remain grouped together to allow efficient recycling.

Hell and colleagues found that the fluorescently tagged synaptic vesicle membrane protein synaptotagmin remains clustered on the presynaptic membrane during the recycling process, rather than being dispersed across the membrane. The same result was found even following intense stimulation of the nerve terminal. This finding suggests that the vesicles remain as an entity throughout the recycling process, although further studies are needed to determine whether all synaptic vesicle proteins remain clustered in this way after exocytosis.

This work not only provides compelling evidence about the mechanisms of synaptic vesicle recycling, but also demonstrates the potential of STED as a powerful new tool that could be used to address other questions in neuroscience relating to cellular structures at the nanoscale level - possibly at even higher resolutions than seen here.

Alison Rowan

ORIGINAL RESEARCH PAPER Willig, K. I. et al. STED microscopy reveals that synaptotagmin remains clustered after synaptic vesicle exocytosis. Nature 440, 935-939 (2006) FURTHER READING Simpson, G. J. The diffraction barrier broken. Nature 440, 879-880 (2006) 